\title{
Characterization of coherent structures in dissipative systems using nonlinear Fourier transform
}

Chekhovskoy, I., Shtyrina, O., Fedoruk, M., Medvedev, S., Turitsyn, S.

I. S. Chekhovskoy, O. V. Shtyrina, M. P. Fedoruk, S. B. Medvedev, S. K. Turitsyn, "Characterization of coherent structures in dissipative systems using nonlinear Fourier transform," Proc. SPIE 11358, Nonlinear Optics and its Applications 2020, 113581B (1 April 2020); doi: 10.1117/12.2555076

SPIE. Event: SPIE Photonics Europe, 2020, Online Only 


\title{
Characterization of coherent structures in dissipative systems using nonlinear Fourier transform
}

\author{
I. S. Chekhovskoy ${ }^{\mathrm{a}, \mathrm{b}}$, O. V. Shtyrina ${ }^{\mathrm{a}, \mathrm{b}}$, M. P. Fedoruk ${ }^{\mathrm{a}, \mathrm{b}}$, S. B. Medvedev ${ }^{\mathrm{a}, \mathrm{b}}$, and \\ S. K. Turitsyn ${ }^{\mathrm{a}, \mathrm{c}}$ \\ ${ }^{a}$ Novosibirsk State University, Novosibirsk 630090, Russia \\ ${ }^{b}$ Institute of Computational Technologies, SB RAS, Novosibirsk 630090, Russia \\ ${ }^{c}$ Aston Institute of Photonic Technologies, Aston University, Birmingham B4 7ET, UK
}

\begin{abstract}
We demonstrated how the nonlinear Fourier transform based on the Zakharov-Shabat spectral problem can be used to characterise coherent structures in dissipative systems. We consider as a particular, albeit important practical example model equation that is widely used to analyse laser radiation and demonstrate that dissipative solitons can be described by a limited number of degrees of freedom - discrete eigenvalues. Our approach can be applied for signal processing in a number of optical systems, from lasers to micro-resonators.
\end{abstract}

Keywords: Nonlinear Fourier transform, nonlinear Schrödinger equation, cubic Ginzburg-Landau equation, numerical simulations, dissipative systems

\section{INTRODUCTION}

The Fourier transform is arguably one of the most important mathematical methods used in engineering. It is widely applied in science and technology and is embedded as an inherent part in numerous signal processing techniques. In conventional engineering applications, the Fourier transform has two major purposes: first, to decompose a complex signal into its more simple constituents, e.g. present temporal signal in the basis of spectral components, improving overall understanding of the signal; second, it can facilitate analysis, e.g. allow us to solve certain type of equations when evolution in the spectral presentation is more simple compared to time domain. Here we discuss a new engineering signal processing concept for the analysis of localised structures. We will focus on the optical context, however, the concept is much broader and can be applied across areas of science and engineering.

We examine new applications of the nonlinear Fourier Transform (NFT) (also known as the inverse scattering method), that can be considered as a generalization of the standard linear Fourier transform to the case of nonlinear systems. NFT was introduced ${ }^{1-5}$ as a powerful mathematical method to solve nonlinear partial differential equations - integrable nonlinear equations. In particular, NFT based on the Zakharov-Shabat spectral problem (that will be described below) allows one to solve the nonlinear Schrödinger equation (NLSE) ${ }^{2}$ - one of the seminal nonlinear models with a range of applications across various fields of science.

The NFT based on the Zakharov-Shabat spectral problem makes it possible to simplify the analysis of the NLSE and reduce complex nonlinear dynamics to simple evolution in a certain basis - the so-called nonlinear spectrum that consists of a continuous and discrete part. Recently we have introduced a concept of using NFT for analysis of the evolution of dissipative, non-integrable systems, ${ }^{6,7}$ see also. ${ }^{8-10}$ Nonlinear cubic GinzburgLandau equation (CGLE), that is a particularly important example in the context of modeling laser systems was considered in. ${ }^{6}$ We have demonstrated that the optical pulse dynamics in the framework of CGLE can be

Further author information: (Send correspondence to I.S.C.)

I.S.C.: E-mail: i.s.chekhovskoy@nsu.ru

O.V.S.: E-mail: olya.shtyrina@gmail.com

M.P.F.: E-mail: mifester@gmail.com

S.B.M.: E-mail: medvedev@ict.nsc.ru

S.K.T.: E-mail: s.k.turitsyn@aston.ac.uk

Nonlinear Optics and its Applications 2020, edited by Neil G. R. Broderick, John M. Dudley, Anna C. Peacock, Proc. of SPIE Vol. 11358, 113581B · (C) 2020 SPIE · CCC code: 0277-786X/20/\$21 - doi: 10.1117/12.2555076 
described by a limited number of discrete eigenvalues of the corresponding Zakharov-Shabat spectral problem. Even though CGLE is not the integrable system, we can still formally compute the nonlinear spectrum of the optical field at each point during propagation and, thus, study the evolution of the nonlinear spectrum. It was demonstrated, that under certain conditions, the NFT may allow facilitating analysis of the evolution of coherent structures with a relatively small number of parameters.

In the current study, we present new results on the application of this approach. To demonstrate that the NFT based signal processing is not strongly linked to the original NLSE, we consider here both anomalous and normal dispersion in the CGLE. For both signs of dispersion, stationary solutions in the form of chirped dissipative solitons are studied. We examine the dependence of their nonlinear spectrum on the amplitude, width, and chirp, and the ratio of the energy associated with the discrete spectrum to the total energy. We anticipate that when most of the energy is in the discrete eigenvalues, those stationary regimes that can be described with high accuracy only by the discrete component of the nonlinear spectrum.

We present here several examples of the solutions of the CGLE with normal and anomalous dispersion when the fraction of energy associated with the discrete spectrum is above $95 \%$ of the total energy. The evolution of the discrete spectrum is calculated using the NFT, and the optical field in the temporal domain was restored from the nonlinear spectrum using the method based on the Darboux transformation. A comparison of the initial and reconstructed optical fields shows good agreement, indicating a potential of presenting complex nonlinear temporal dynamics by the evolution of a small number of degrees of freedom.

\section{MATHEMATICAL MODEL}

Similar to the analysis presented in,${ }^{6}$ we examine here a new application of the inverse scattering transform, ${ }^{1-5,11}$ also known as the nonlinear Fourier transform, originally developed for the nonlinear Schrödinger equation $(\mathrm{NLSE}):^{2}$

$$
i \frac{\partial U}{\partial z}+\frac{1}{2} \frac{\partial^{2} U}{\partial t^{2}}+|U|^{2} U=0
$$

The Zakharov-Shabat problem (ZSP) for the signal $U(z, t)$ can be written as

$$
\left\{\begin{array}{l}
\frac{\partial \psi_{1}}{\partial t}=-i \zeta \psi_{1}+U(z, t) \psi_{2} \\
\frac{\partial \psi_{2}}{\partial t}=-U^{*}(z, t) \psi_{1}+i \zeta \psi_{2}
\end{array}\right.
$$

Here the spectral parameter is $\zeta=\xi+i \eta$. The spectrum of ZSP (2) consists of two parts:

(i) a continuous spectrum that is defined on the real axis of the complex plane $\zeta=\xi$ by the complex function $r(\xi)$,

(ii) a discrete spectrum that is given by $4 \times N$ real parameters (the set of complex-valued eigenvalues $\left\{\zeta_{n}\right\}$ having a positive imaginary part together with complex-valued norming constants $\left\{r_{n}\right\}$ ).

The discrete eigenvalues determine a soliton content of the field distribution, with $N$ being the total number of solitons in the propagating field $U(z, t)$. The discrete nonlinear spectrum defines the soliton content of the field $U(z, t)$. When $U(z, t)$ represents a set of well-separated solitons, each eigenvalue $\zeta_{n}$ specifies parameters of individual soliton: the amplitude $2 \operatorname{Im}\left(\zeta_{n}\right)$, the frequency $-2 \operatorname{Re}\left(\zeta_{n}\right)$, the position $T_{n}=\log \left[\left|r_{n}\right| /\left(2 \operatorname{Im} \zeta_{n}\right)\right] /\left(2 \operatorname{Im} \zeta_{n}\right)$, and the phase $\varphi_{n}=-\arg \left(i r_{n}\right)$. The total energy of the solution of NLSE can be presented as a sum of the continuous and discrete spectra:

$$
E_{t}=\int_{-\infty}^{\infty}|U(z, t)|^{2} d t=\sum_{n=1}^{N} 4 \eta_{n}+\frac{1}{\pi} \int_{-\infty}^{\infty} \log \left(1+|r(\xi)|^{2}\right) d \xi=E_{d}+E_{c}
$$

Consider now an optical field evolving according to the CGLE, having the following form:

$$
i \frac{\partial U}{\partial z}+\frac{s}{2} \frac{\partial^{2}}{\partial t^{2}} U+|U|^{2} U=i\left(\sigma+\alpha \frac{\partial^{2}}{\partial t^{2}}+\delta|U|^{2}\right) U,
$$


where $s=-\operatorname{sign}\left(\beta_{2}\right)$, the parameter $\delta>0$ corresponds to the effective distributed saturable absorber action and the parameter $\alpha>0$ describes the effect of distributed optical filtering. The saturated gain $g_{0}$ and linear loss $\Gamma$ define the parameter $\sigma$ :

$$
\sigma(E)=\frac{g_{0}}{2\left(1+E / E_{\text {sat }}\right)}-\frac{\Gamma}{2}, \quad E(z)=\int|U(z, t)|^{2} d t .
$$

The CGL equation has special solutions in the form of chirped dissipative solitons

$$
U(z, t)=U_{0}^{1+i C}(t) \exp \{i \phi z\}, \quad U_{0}(t)=\frac{A}{\cosh (t / \tau)}
$$

The CGL equation (4) is not integrable, however, we can still formally compute the NFT (nonlinear) spectrum of the optical field $U(z, t)$ at each point $z$ and consider the evolution of the nonlinear spectrum with $z\left(\operatorname{see~also~}^{12}\right)$. The Zakharov-Shabat problem (2) is solved here numerically using the Boffetta-Osborne method. ${ }^{13}$ A combined method is used to localize discrete eigenvalues, including the preliminary finding of the zeros of the function $a(\zeta)$ using contour integrals, and their subsequent refinement using the Newton method $^{14}$ and the exponential 4 th order scheme. ${ }^{15}$

The application of the NFT-based analysis to the general chirped pulse given by (6) with independent parameters $A, \tau, C$ gives the expression ${ }^{16-20}$ for the discrete spectrum size $N: A^{2} \tau^{2}=C^{2} / 4+(N-1 / 2)^{2}$. The set of eigenvalues $\left\{\zeta_{n}\right\}$ of such pulses is defined as $\zeta_{n}=i \eta_{n}=i\left(\sqrt{A^{2} \tau^{2}-C^{2} / 4}+1 / 2-n\right)$, where $n$ are positive integers satisfying condition $\sqrt{A^{2} \tau^{2}-C^{2} / 4}+1 / 2-n>0$, providing for $\operatorname{Im}\left(\zeta_{n}\right)>0$. Previously we derived the analytical expression for the fraction of energy containing in the discrete spectrum:

$$
\frac{E_{d}}{E_{t}}=\frac{A^{2} \tau^{2}-C^{2} / 4-(\rho-1 / 2)^{2}}{A^{2} \tau^{2}}
$$

where $\rho=\operatorname{frac}\left[\sqrt{A^{2} \tau^{2}-C^{2} / 4}+1 / 2\right]$ is a fractional part of $\sqrt{A^{2} \tau^{2}-C^{2} / 4}+1 / 2$.

Next, we restrict the family of pulses (6) to the stationary solutions of the CGLE making the parameters $A$, $C$ and $\tau$ dependent and defined by $\delta, \alpha, g_{0}, \Gamma$ and $E_{\text {sat }}$. For $\delta>0$ and $\alpha>0$ chirp parameter $C$ is given by the expression

$$
C=-\frac{3}{2} \frac{(\delta \alpha+s / 2)}{(-\delta s / 2+\alpha)} \pm \frac{1}{2} \sqrt{9 \frac{(\delta \alpha+s / 2)^{2}}{(-\delta s / 2+\alpha)^{2}}+8}
$$

Here the sign in the above equation is determined from the condition $C /(-\delta / 2+\alpha)>0$. It is convenient to denote $\mathrm{R}(C, \alpha)=\alpha\left(C^{2}-1\right)+s C$ and $\mathrm{B}(C, \alpha)=3 \alpha C+\frac{s}{2}\left(C^{2}-2\right)$, then remaining dissipative soliton parameters are given by

$$
\begin{aligned}
A & =\sqrt{\sigma \mathrm{B}(C, \alpha) / \mathrm{R}(C, \alpha)} \\
\tau & =\sqrt{\mathrm{R}(C, \alpha) / \sigma} \\
\phi & =\sigma\left(2 \alpha C-\left(C^{2}-1\right) \frac{s}{2}\right) / \mathrm{R}(C, \alpha) .
\end{aligned}
$$

The energy $E_{s}$ of the steady-state solution is expressed either as

$$
E_{s}=2 A^{2} \tau=2 \mathrm{~B}(C, \alpha) \sqrt{\sigma / \mathrm{R}(C, \alpha)}
$$

or as $E_{s}=E_{\text {sat }}\left(g_{0} /(2 \sigma+\Gamma)-1\right)$, see (5). After energy $E_{s}$ is found, it fully determines parameter $\sigma$, sign of which will be important in the following analysis.

The value of the gain coefficient $\sigma$ for stationary solutions can be found analytically using the definition of $\sigma(5)$ and the asymptotic value of the energy $E_{s}(10)$. So we need to solve the following equation in the case of $g_{0}>\Gamma$ and get $\sigma \in\left(-\Gamma / 2,\left(g_{0}-\Gamma\right) / 2\right)$ :

$$
E_{\text {sat }}\left(\frac{g_{0}}{2 \sigma+\Gamma}-1\right)=2 \mathrm{~B}(C) \sqrt{\frac{\sigma}{\mathrm{R}(C)}} .
$$

We consider now the possible choices of parameters and corresponding solutions. 
$\mathbf{R}(C)<0 \Rightarrow \sigma \in(-\Gamma / 2,0):$

$$
E_{\text {sat }}\left(\frac{g_{0}}{-2(\sqrt{-\sigma})^{2}+\Gamma}-1\right)=\frac{2 \mathrm{~B}(C)}{\sqrt{-\mathrm{R}(C)}} \sqrt{-\sigma}=\Sigma_{-} \times \sqrt{-\sigma} .
$$

Let denote

$$
Q=\frac{1}{9}\left(\frac{E_{\text {sat }}^{2}}{\Sigma_{-}^{2}}+\frac{3 \Gamma}{2}\right), \quad R=\frac{E_{\text {sat }}}{\Sigma_{-}}\left(\frac{Q}{3}+\frac{g_{0}}{4}-\frac{2 \Gamma}{9}\right) .
$$

When $R^{2} \leq Q^{3}$ the Eq. (12) has two solutions in the required interval for the strict inequality or only one for the equality. The corresponding roots read:

$$
\sqrt{-\sigma_{ \pm}}=-2 \sqrt{Q} \cos \left(\frac{1}{3} \arccos \frac{R}{\sqrt{Q^{3}}} \pm \frac{2 \pi}{3}\right)-\frac{E_{\text {sat }}}{3 \Sigma_{-}}
$$

it is easy to see that $-\sigma_{+}>-\sigma_{-}$, which leads to $E\left(\sigma_{+}\right)>E\left(\sigma_{-}\right)$. However, the solution with $\sigma_{+}$is unstable, therefore, in the following, it will not be considered.

$\mathbf{R}(C)=0 \Rightarrow \sigma=0:$

$$
E_{s}=E_{\text {sat }} \times\left(g_{0}-\Gamma\right) / \Gamma \quad \Rightarrow \quad \tau=\frac{2 \mathrm{~B}(C)}{E_{s}}
$$

$\mathbf{R}(C)>0 \Rightarrow \sigma \in\left(0,\left(g_{0}-\Gamma\right) / 2\right):$

$$
E_{\text {sat }}\left(\frac{g_{0}}{2(\sqrt{\sigma})^{2}+\Gamma}-1\right)=\frac{2 \mathrm{~B}(C)}{\sqrt{\mathrm{R}(C)}} \sqrt{\sigma}=\Sigma_{+} \times \sqrt{\sigma} .
$$

However, we note that stationary solutions with $\sigma>0$ are unstable. To make classification of solutions, denote:

$$
Q=\frac{1}{9}\left(\frac{E_{s a t}^{2}}{\Sigma_{+}^{2}}-\frac{3 \Gamma}{2}\right), \quad S=\frac{E_{s a t}}{\Sigma_{+}}\left(\frac{Q}{3}-\frac{g_{0}}{4}+\frac{2 \Gamma}{9}\right) .
$$

Now the families of solutions read: If $S^{2} \leq Q^{3}$, then

$$
\sqrt{\sigma}=-2 \sqrt{Q} \cos \left(\frac{1}{3} \arccos \frac{S}{\sqrt{Q^{3}}}\right)-\frac{E_{s a t}}{3 \Sigma_{+}}
$$

If $S^{2}>Q^{3}>0$, then

$$
\sqrt{\sigma}=-2 \operatorname{sign}(S) \sqrt{Q} \cosh \left(\frac{1}{3} \operatorname{arccosh} \frac{|S|}{\sqrt{Q^{3}}}\right)-\frac{E_{\text {sat }}}{3 \Sigma_{+}}
$$

If $S^{2}>0>Q^{3}$, then

$$
\sqrt{\sigma}=-2 \operatorname{sign}(S) \sqrt{|Q|} \sinh \left(\frac{1}{3} \operatorname{arcsinh} \frac{|S|}{\sqrt{|Q|^{3}}}\right)-\frac{E_{\text {sat }}}{3 \Sigma_{+}}
$$

If $Q=0$, then

$$
\sqrt{\sigma}=\left(\frac{E_{\text {sat }}}{\Sigma_{+}} \frac{\left(g_{0}-\Gamma\right)}{2}+\left(\frac{E_{\text {sat }}}{3 \Sigma_{+}}\right)^{3}\right)^{1 / 3}-\frac{E_{\text {sat }}}{3 \Sigma_{+}} .
$$

The existence domain of the CGLE chirped solitons is determined only by the choice of the gain $g_{0}, \Gamma$ and $E_{\text {sat }}$ parameters and it can be asymptotically expanded to the quadrant $\delta \geq 0, \alpha \geq 0$ with $E_{\text {sat }} \times\left(g_{0}-\Gamma\right) / \Gamma \rightarrow 0$, which is illustrated by Fig. 1 . Here we considered the parameters $g_{0}=0.3, \Gamma=0.1, E_{\text {sat }}=1$ (which give the energy value $E_{s}=E_{s a t} \times\left(g_{0}-\Gamma\right) / \Gamma=2$ at $\left.\delta=\alpha=0\right)$. First, we computed the dependence of the characteristics of the nonlinear spectrum of the CGLE stationary solutions (4) on the parameters $\alpha$ and $\delta$, with all others fixed as described above. Figure 1 present the ratio of the energy of the discrete part of the nonlinear 

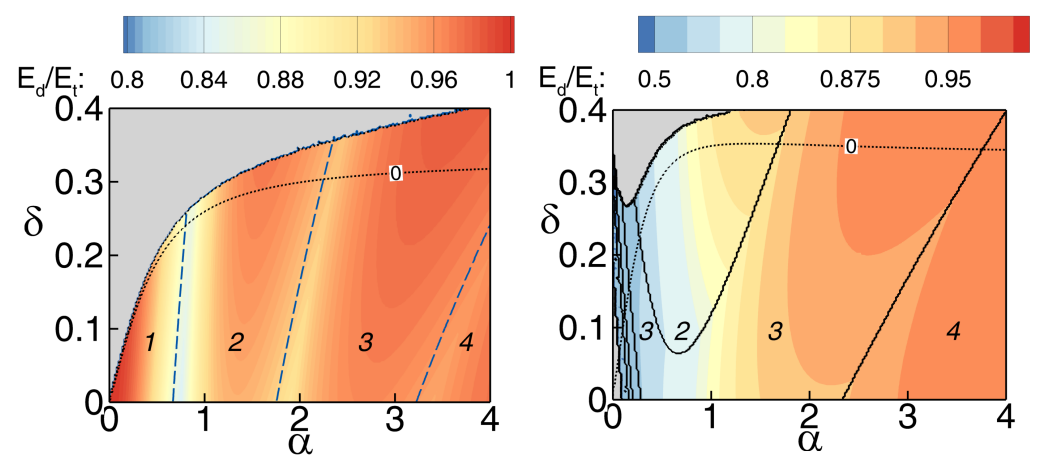

Figure 1. Dependence of the fraction of the energy in the discrete spectrum in the total energy $E_{d} / E_{t}$, on the parameters $\alpha$ and $\delta$ for $g_{0}=0.3, \Gamma=0.1$ in the case of anomalous dispersion (left) and normal dispersion (right). The numbers of black colour in the figure indicate the number of discrete levels for the corresponding CGLE stationary solutions (6), and the black lines separate the areas with different eigenvalue counts. The dashed line indicates the level $\sigma=0$ dividing stable and unstable stationary solutions.

spectrum $E_{d}$, to the total energy $E_{t}$ as a function of the parameters $\alpha$ and $\delta$ for both signs of dispersion. For each pair of parameters, continuous and discrete ZSP spectra have been numerically found for the corresponding stationary solution of the CGLE (6). The number of discrete eigenvalues for each set of parameters is plotted in the corresponding areas in Fig. 1. One can see that the proportion of the energy containing in the discrete spectrum to the total energy is always quite high - more than $82 \%$. However, the areas with 1 and 3 discrete eigenvalues have sub-domains, where the energy ratio exceeds $97.5 \%$. This indicates that dynamics is dominated by the coherent structures and NFT might be the appropriate way to reduce an effective number of the degrees of freedom required for the description of this system.

The boundaries separating areas with different discrete eigenvalue count (from 1 to 4 ) obtained numerically are in full agreement with the analytical formulae given the numbers of discrete eigenvalues $N$.

\section{NFT-BASED ANALYSIS OF THE DYNAMICS OF DISSIPATIVE SOLITONS}

We now examine with more details application of the NFT for analysis of CGLE (4) dynamics with anomalous and normal dispersion. Numerical modelling was performed using the standard split-step Fourier method (SSFM). We consider as the initial condition the NLSE soliton in the form

$$
U(z=0, t)=0.2 \operatorname{sech}(0.2 t)
$$

with the peak power, that is less than the power of the stationary solutions of the CGLE (6) with the given parameters $\alpha$ and $\delta$.
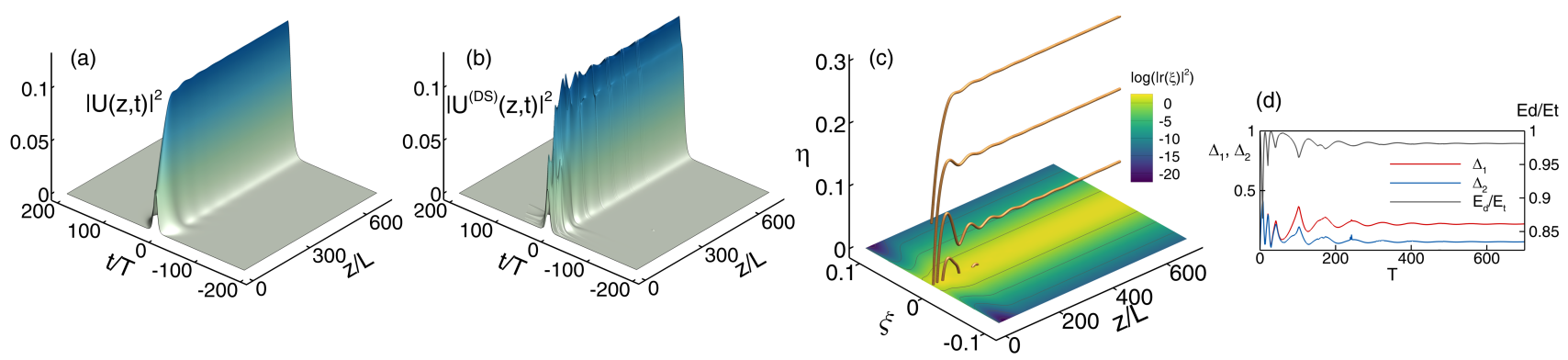

Figure 2. Anomalous dispersion, $\alpha=3.5, \delta=0.35(\sigma<0)$. (a) original pulse intensity dynamics $U(z, t),(\mathrm{b})$ reconstructed from the discrete spectrum field $U^{(D S)}(z, t)$, (c) the discrete spectrum (upper part) and the logarithm of $|r(\xi)|^{2}$ for continuous spectrum (counterplot), (d) the ratio $E_{d} / E_{t}$ and the relative $L_{2}$-norm of the difference between original field $U(z, t)$ and reconstructed field $U^{(D S)}(z, t)$ (the quantity $\left.\Delta_{1}\right)$ and between the amplitudes $|U(z, t)|$ and $\left|U^{D S}(z, t)\right|($ the quantity $\left.\Delta_{2}\right)$. 

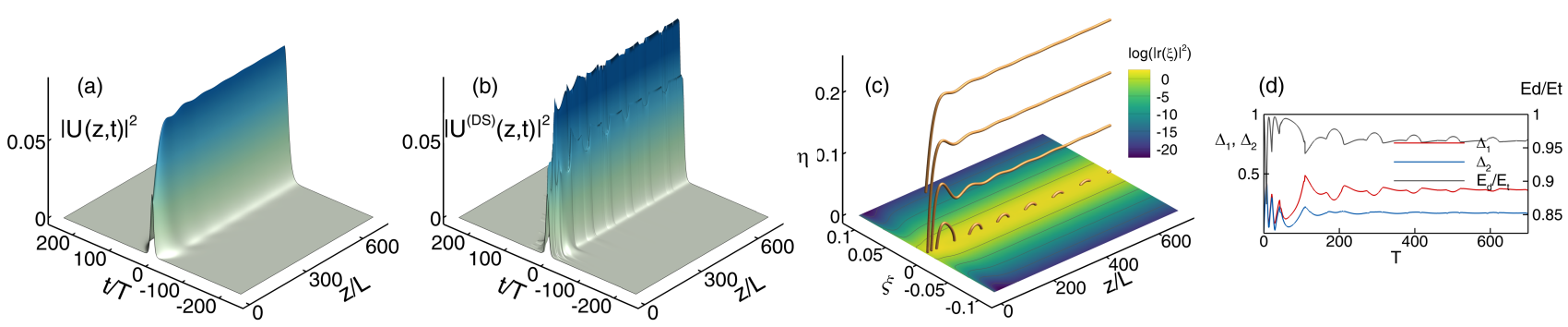

Figure 3. Normal dispersion, $\alpha=3.5, \delta=0.35(\sigma<0)$. (a) original pulse intensity dynamics $U(z, t)$, (b) reconstructed from the discrete spectrum field $U^{(D S)}(z, t)$, (c) the discrete spectrum (upper part) and the logarithm of $|r(\xi)|^{2}$ for continuous spectrum (counterplot), (d) the ratio $E_{d} / E_{t}$ and the relative $L_{2}$-norm of the difference between original field $U(z, t)$ and reconstructed field $U^{(D S)}(z, t)$ (the quantity $\Delta_{1}$ ) and between the amplitudes $|U(z, t)|$ and $\left|U^{D S}(z, t)\right|($ the quantity $\left.\Delta_{2}\right)$.

The initial field always has only a single discrete eigenvalue, and the peak power of this field increases with propagation along $z$. We consider two situations: one with unstable dynamics with $\sigma>0$ when the energy dissipates, and one with stable propagation with $\sigma<0$, when initial signal approximates to the steady-state soliton regime. For each of these examples, the nonlinear spectrum was calculated at 500 points along $z$ for $3 \mathrm{~d}$ field dynamics figures and at 5000 points for the discrete spectrum evolution figures.

Figures. 2 and 3 shows stable dynamics along $z$ of the field intensity and the dynamics of discrete eigenvalues $\zeta_{n}=\xi_{n}+\eta_{n}$ in the case of $\alpha=3.5, \delta=0.35, \sigma<0$ for anomalous and normal dispersion, respectively. The initial single discrete eigenvalue evolves into three clearly seen discrete eigenvalues with that correspond to the discrete NFT spectrum of the chirped CGLE soliton (6) for the considered values of the parameters. In the case of anomalous dispersion oscillations that appear at first quickly fade out, and the fourth discrete eigenvalue that appeared at the beginning disappears. The fourth discrete eigenvalue in the case of normal dispersion periodically exists, but its imaginary part decreases. The important observation is that the major part of the energy is in the discrete spectrum, making NFT an attractive approach to reduce the number of degrees of freedom, even analyzing such unstable dynamics. In both cases the ratio $E_{d} / E_{t}$ of energies is high, and does not become less $85 \%$, even at the transient regime. The asymptotic value of the fraction of discrete spectrum energy $E_{d}$ is close to $97 \%$ for anomalous dispersion case, and $96 \%$ for normal dispersion. When the fourth discrete eigenvalue in the last case appears, it pulls the energy from the continuous spectrum, so the ratio $E_{d} / E_{t}$ temporarily increases. The field $U^{(D S)}(z, t)$, which is reconstructed from the discrete spectrum, coincides qualitatively with original

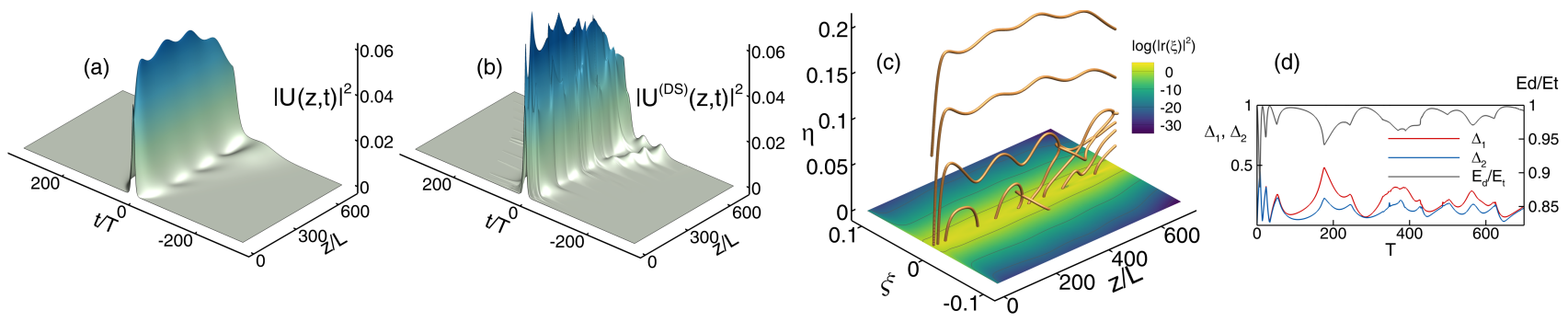

Figure 4. Anomalous dispersion, $\alpha=3.5, \delta=0.35(\sigma>0)$. (a) original pulse intensity dynamics $U(z, t)$, (b) reconstructed from the discrete spectrum field $U^{(D S)}(z, t)$, (c) the discrete spectrum (upper part) and the logarithm of $|r(\xi)|^{2}$ for continuous spectrum (counterplot), (d) the ratio $E_{d} / E_{t}$ and the relative $L_{2}$-norm of the difference between original field $U(z, t)$ and reconstructed field $U^{(D S)}(z, t)$ (the quantity $\Delta_{1}$ ) and between the amplitudes $|U(z, t)|$ and $\left|U^{D S}(z, t)\right|($ the quantity $\left.\Delta_{2}\right)$. 


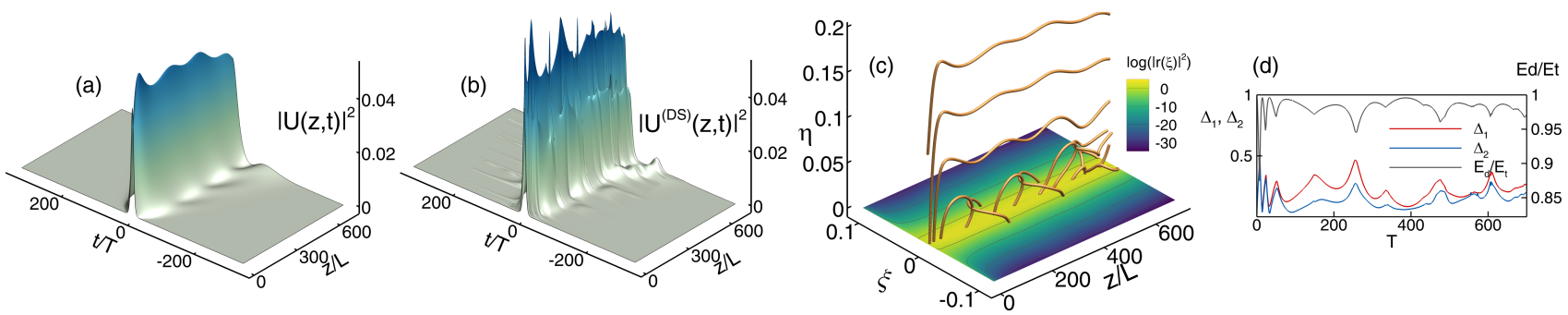

Figure 5. Normal dispersion, $\alpha=3.5, \delta=0.35(\sigma>0)$. (a) original pulse intensity dynamics $U(z, t)$, (b) reconstructed from the discrete spectrum field $U^{(D S)}(z, t)$, (c) the discrete spectrum (upper part) and the logarithm of $|r(\xi)|^{2}$ for continuous spectrum (counterplot), (d) the ratio $E_{d} / E_{t}$ and the relative $L_{2}$-norm of the difference between original field $U(z, t)$ and reconstructed field $U^{(D S)}(z, t)$ (the quantity $\Delta_{1}$ ) and between the amplitudes $|U(z, t)|$ and $\left|U^{D S}(z, t)\right|($ the quantity $\Delta_{2}$ ).

field $|U(z, t)|$ (see Figs. $2 \mathrm{~b}$ and $3 \mathrm{~b}$ ), and the relative $L_{2}$-norm of the difference between them

$$
\Delta_{1}=\sqrt{\frac{\sum_{j=0}^{M}\left|U\left(z, t_{j}\right)-U^{(D S)}\left(z, t_{j}\right)\right|^{2}}{\sum_{j=0}^{M}\left|U\left(z, t_{j}\right)\right|^{2}}},
$$

reaches the value $\Delta_{1}=0.22\left(\Delta_{2}=0.07\right.$ for the absolute values of the fields $)$ at $z=700$ in the case of anomalous dispersion, but growth up to $\Delta_{1}=0.36\left(\Delta_{2}=0.17\right)$ for the normal dispersion.

Figures. 4 and 5 corresponds to the unstable case $(\sigma>0)$. Oscillations of the field intensity distribution are accompanied by the spatial variations of the discrete spectrum. Again, it can be seen that, from one initial discrete eigenvalue, the discrete spectrum is growing into several eigenvalues that contain most of the field energy (more than 95\%). Two eigenvalues evolve almost stably with $z$ for the anomalous dispersion, and three eigenvalues for the normal dispersion. Two others appear and decay periodically with propagation. The periodically arising fourth discrete eigenvalue merges with the closest discrete point in the NFT spectrum, leading to quasi-symmetric configuration of these two eigenvalues relative to the real axis and out-of phase oscillations. The relative $L_{2}$-norm of the difference between original and reconstructed fields $\Delta_{1}$ and field absolute values $\Delta_{2}$ do not differ from each other not so significantly in these examples compared with previous examples. Moreover, at $z=700$ these errors reaches values $\Delta_{1}=0.15, \Delta_{2}=0.14$ (anomalous dispersion), and $\Delta_{1}=0.26, \Delta_{2}=0.20$ (normal dispersion).

\section{ACKNOWLEDGMENTS}

This work was supported by the Russian Science Foundation (grant No. 17-72-30006).

\section{REFERENCES}

[1] Gardner, C. S., Greene, J. M., Kruskal, M. D., and Miura, R. M., "Method for Solving the Korteweg-deVries Equation," Physical Review Letters 19, 1095-1097 (11 1967).

[2] Zakharov, V. E. and Shabat, A. B., "Exact Theory of Two-Dimensional Self-Focusing and One-Dimensional Self-Modulation of Waves in Non-Linear Media," Journal of Experimental and Theoretical Physics 34(1), 62-69 (1972).

[3] Ablowitz, M. J., Kaup, D. J., Newell, A. C., and Segur, H., "The Inverse Scattering Transform-Fourier Analysis for Nonlinear Problems," Studies in Applied Mathematics 53, 249-315 (12 1974).

[4] G. L. Lamb Jr., [Elements of Soliton Theory], John Wiley \& Sons, New York (1980).

[5] Ablowitz, M. J. and Segur, H., [Solitons and the Inverse Scattering Transform], Society for Industrial and Applied Mathematics, Philadelphia (1981). 
[6] Chekhovskoy, I. S., Shtyrina, O. V., Fedoruk, M. P., Medvedev, S. B., and Turitsyn, S. K., "Nonlinear Fourier Transform for Analysis of Coherent Structures in Dissipative Systems," Physical Review Letters 122, 153901 (4 2019).

[7] Sugavanam, S., Kopae, M. K., Peng, J., Prilepsky, J. E., and Turitsyn, S. K., "Analysis of laser radiation using the nonlinear fourier transform," Nature Communications 10, 5663 (2019).

[8] Sugavanam, S., Kamalian, M., Perego, A. M., Prilepsky, J. E., and Turitsyn, S. K., "Experimentally characterized nonlinear fourier transform of a mode-locked fibre laser," in [In 2017 Conference on Lasers and Electro-Optics Europe 83 European Quantum Electronics Conference (CLEO/Europe-EQEC, IEEE, New York)], 472, -11 (2017).

[9] Ryczkowski, P., Närhi, M., Billet, C., Merolla, J.-M., Genty, G., and Dudley, J. M., "Real-time full-field characterization of transient dissipative soliton dynamics in a mode-locked laser," Nature Photonics 12(4), 221 (2018).

[10] Osborne, A. R., "Nonlinear fourier methods for ocean waves," Procedia IUTAM 26, 112-123 (2018).

[11] Turitsyn, S. K., Prilepsky, J. E., Le, S. T., Wahls, S., Frumin, L. L., Kamalian, M., and Derevyanko, S. A., "Nonlinear Fourier transform for optical data processing and transmission: advances and perspectives," Optica 4(3), 307 (2017).

[12] Prilepsky, J. E., Derevyanko, S. A., and Turitsyn, S. K., "Conversion of a chirped Gaussian pulse to a soliton or a bound multisoliton state in quasi-lossless and lossy optical fiber spans," Journal of the Optical Society of America B 24, 1254 (6 2007).

[13] Boffetta, G. and Osborne, A., "Computation of the direct scattering transform for the nonlinear Schroedinger equation," Journal of Computational Physics 102, 252-264 (10 1992).

[14] Vasylchenkova, A., Prilepsky, J. E., and Turitsyn, S. K., "Contour integrals for numerical computation of discrete eigenvalues in the Zakharov-Shabat problem," Optics Letters 43(15), 3690 (2018).

[15] Medvedev, S., Vaseva, I., Chekhovskoy, I., and Fedoruk, M., "Exponential fourth order schemes for direct Zakharov-Shabat problem," Optics Express 28, 20 (1 2020).

[16] Satsuma, J. and Yajima, N., "B. Initial Value Problems of One-Dimensional Self-Modulation of Nonlinear Waves in Dispersive Media," Progress of Theoretical Physics Supplement 55, 284-306 (1974).

[17] Grunbaum, F. A., "The scattering problem for a phase-modulated hyperbolic secant pulse," Inverse Problems 5(3), 287-292 (1989).

[18] Tovbis, A. and Venakides, S., "The eigenvalue problem for the focusing nonlinear Schrödinger equation: new solvable cases," Physica D: Nonlinear Phenomena 146, 150-164 (11 2000).

[19] Desaix, M., Helczynski, L., Anderson, D., and Lisak, M., "Propagation properties of chirped soliton pulses in optical nonlinear Kerr media," Physical Review E 65, 056602 (4 2002).

[20] Bambini, A. and Berman, P. R., "Analytic solutions to the two-state problem for a class of coupling potentials," Physical Review A 23, 2496-2501 (5 1981). 